\section{Hilfreiche Inhalte für die Praxis-Website}

Mit diesen Website-Inhalten punkten Praxen:

—-Sprechzeiten
_-Leistungsspektrum
_-Hinweis auf Notdienst
_-Anamnese- und Aufklärungsbögen
zum Download
_-Veranstaltungshinweise
(z.B. zu Schulungen, Kursen)
_-Videos, Podcasts und Grafiken zu
Gesundheitsthemen

\section{Daten und Fakten: 2000}

Mit Kosten von 1.500 bis $2.000 €$ müssen Ärzte für eine professionell gestaltete Website rechnen, so die Erfahrung der Ärztin und Webdesignerin Dr. Christine Trutt-lbing. Damit habe die Praxis dann einen Webauftritt mit zehn bis zwölf Unterseiten und professionell geschossenen Fotos. Und gerade auf Letzteres sollten die Praxen wert legen. „Das ist Ihr Aushängeschild im Internet", so TruttIbing. Viele Webbesucher würden von der Bildqualität auf die Kompetenz des Arztes schließen. Für Schmuckbilder können Praxen auf Online-Fotoagenturen wir Fotolia oder Pixelio zurückgreifen.

\section{So kommt der Webauftritt auch in Suchmaschinen gut an}

Wer bei Suchmaschinen wie Google in den oberen Rängen gelistet werden will, sollte regelmäßig Inhalte aktualisieren, diese ordentlich in Text und Überschriften strukturieren und darauf achten, dass jede Unterseite einen guten Seitentitel hat (im Reiter oben in der Website zu sehen - etwa "Leistungsspektrum Praxis Muster" für die Unterseite mit dem Leistungsspektrum, oder für Gesundheitsthemen etwa "Akupunktur"). Des Weiteren ist die Verwendung von strategischen Schlagworten empfehlenswert und es sollte für eine gute Verlinkung etwa zu Arztsuchportalen gesorgt werden.

\section{Vorsicht mit Kartenmaterial!}

Die Lage der Praxis auf einer Karte darzustellen, ist ein guter Service. Solche Karten dürfen aber nicht einfach kopiert werden, denn sie sind häufig urheberrechtlich geschützt. Kostenloses Kartenmaterial erhält man unter maps.google.com oder bei www.openstreetmap.de.

Rebekka Höhl bei einem Anbieterwechsel der Name nicht mitnehmen. "Und das ist gerade aus Marketing- und Bekanntheitsgründen unschön.

Die Ärztin und Webdesignerin rät außerdem davon ab, die ganze Aktualisierung der Website in die Hände von Bekannten zu legen. Denn wenn die irgendwann das Interesse verlieren, muss sich das Praxisteam plötzlich allein einarbeiten. Aber auch, wenn man sich für die Website-Pflege einen externen Anbieter sucht, sollte man darauf achten, dass man bei Vertragsende weiß, wie man Inhalte bearbeitet. Deshalb gehört zur Vorbereitung des Webauftritts auch, dass man im Team diskutiert, ob man selbst aktualisieren will und wer sich federführend darum kümmert. Dabei gibt es laut Trutt-Ibing Content Management Systeme (CMS), mit denen sich recht leicht die Inhalte der Website bearbeiten lassen. Einige davon sind sogar kostenlos. Hier sollten Ärzte keine Berührungsängste haben. Allerdings: „Damit die Website dauerhaft und sicher funktioniert, müssen regelmäßige Programm-Updates eingespielt werden." Es gibt auch fertige Website-Baukästen, die bereits ein CMS beinhalten.

\section{Auch an mobile Endgeräte denken}

Der nächste Schritt wäre: „Holen Sie sich Anregungen für Ihre Praxis-Website", so die Webdesignerin. Etwa indem die Ärzte sich Webauftritte anderer Praxen ansehen, aber eben auch ganz gezielt ihre Patienten befragen. Denn genau diese - sowie potenzielle Patienten - sollen die Website ja später nutzen. Außerdem schade es nicht, sich gleich vorab zu überlegen, ob die Website auch für mobile Endgeräte wie Smartphones nutzbar sein soll. „Eine für Smartphones optimierte Website kostet aber natürlich auch mehr."

Für die Inhalte der Website gilt ganz klar: Hier müssen Ärzte das Berufsrecht und das Heilmittelwerbegesetz (HWG) beachten. Ebenso gelte noch immer das Fernbehandlungsverbot. Vorsichtig sollten Ärzte auch mit Patientendiskussionsforen sein. „Als Betreiber des Forums ist der Arzt für alles, was im Forum steht, verantwortlich."Wer seinen Patienten ein Kontaktformular auf der Website anbietet, müsse zudem eine Datenschutzerklärung veröffentlichen, über die die Nutzer er- fahren, wie mit ihren Daten verfahren wird und wie diese gespeichert werden.

Ansonsten dürfen Praxen schon sehr viel beim Online-Marketing. Sie können und sollten ihr Leistungsspektrum sowie die Zugehörigkeit zu Praxisverbünden angeben, aber etwa auch sachliche Informationen zu Krankheitsbildern, Untersuchungsmethoden und anderen Gesundheitsthemen im Web veröffentlichen. Letzteres ist laut Trutt-Ibing sogar nahezu ein Muss, wenn man in Suchmaschinen wie Google regelmäßig in oberer Position gelistet werden will - sofern die Infos auch regelmäßig aktualisiert werden. Und natürlich sollten Ärzte Links zu Bewertungsportalen, in denen sie sich angemeldet haben, oder zu Twitter einbinden. Umfangreiche Websites sollten zudem ein Suchfeld haben. „Kleinere Websites mit vier, fünf Unterseiten brauchen das nicht.“

Rebekka Höhl

\section{Wichtige Links}

— Impressumgeneratoren
www.e-recht24.de
www.net-and-law.de
— Content-Management-Systeme
www.joomla.de
typo3.org

— Checklisten für den Webauftritt www.aekn.de/arztspezial/infosfuer-klinik-and-praxis www.cti-webkonzepte.de — Rechtliche Infos www.baek.de (Musterberufsordnung) www.gesetze-im-internet.de.

(Telemediengesetz, Heilmittelwerbegesetz)

Im Rahmen von e.Med bietet Springer Medizin Ärzten mit e.Praxis auch einen Baukasten für die Gestaltung der PraxisWebsite. Mehr dazu finden Sie online unter:

www.springermedizin.de/arztonline/ 